\title{
OPEN Levels and health risk assessment of pesticides and metals in Lycium barbarum L. from different sources in Ningxia, China
}

Yahong Zhang ${ }^{1}$, Jiaqi Oin ${ }^{2}$, Yan Wang ${ }^{2}$, Tongning Zhou ${ }^{2}$, Ningchuan Feng ${ }^{1,3}$, Caihong Ma ${ }^{4}$ \& Meilin Zhu $\mathbf{u}^{1,2,3 凶}$

The berries of Lycium barbarum L. (Goji) are widely used as a Chinese traditional herbal medicine and functional food because of their reported beneficial pharmacological effects. However, there are reports of Goji berries being contaminated by chemical residues that could pose a hazard to humans. In this study, samples of $L$. barbarum $L$. berries were collected from plantations in a genuine production area and supermarkets in Ningxia, China. The major hazardous chemicals, including pesticides (dichlorvos, omethoate, cypermethrin, fenvalerate, malathion, and deltamethrin) and metals (lead (Pb), cadmium (Cd), copper (Cu), nickel (Ni), zinc ( $\mathrm{Zn})$, and arsenic (As)), were quantified by gas chromatography and inductively coupled plasma-optical emission spectrometry. In addition, associated daily exposures and health risks were determined using deterministic and probabilistic assessments. The levels of five pesticides from the plantation samples were considerably lower than the maximum residue limits; only dichlorvos was detected in the supermarket samples, and deltamethrin was not detected in any samples. $\mathrm{Cu}, \mathrm{Zn}, \mathrm{As}, \mathrm{Pb}, \mathrm{Ni}$ and $\mathrm{Cd}$ were detected in samples from both sources. The hazard quotient values of individual hazardous chemicals and the hazard index of combined hazardous chemicals were considerably less than 1, indicating the absence of a non-carcinogenic effect of hazardous chemical exposures through Goji berry consumption. The $R$ value of As was much less than $10^{-6}$, which shows that consumption of the Goji berries had no obvious carcinogenic risks. The potentially harmful effects of the $L$. barbarum $L$. are more likely from berries obtained from plantations than those from supermarkets, and metal exposure is more dangerous than pesticide exposure. However, on the basis of our analysis, no population would be exposed hazardous chemicals exceeding existing standards, and the factors most affecting the health risk were exposure frequency and As content.

Lycium barbarum L. (Solanaceae), produces a fruit known as the Goji berry or wolfberry and has been used as a traditional Chinese medicine for centuries ${ }^{1}$. Goji berries are popular worldwide as a health food, and they are consumed in various forms, including soups, drinks, and in certain dishes ${ }^{2}$ because of their potential beneficial effects. Goji berries are rich in compounds with positive biological activities, including polysaccharides, carotenoids, and flavonoids ${ }^{3}$, and their consumption has been linked with health benefits, such as antioxidant ${ }^{4}$, and anticancer ${ }^{5}$ effects.

Goji berries are mostly cultivated in China. While, there is growing interest in Goji cultivation in other countries, for example, other East Asian countries ( $\operatorname{Japan}^{6}$, South Korea ${ }^{7}$ ), American countries (the USA ${ }^{2}$, Brazil $^{8}$ ), European countries (Italy ${ }^{9}$, Greece $^{10}$, Poland ${ }^{11}$ ) et al. In China, the mainly cultivated areas are in northwestern provinces of China, such as Ningxia, Qinghai, Xinjiang, and Inner Mongolia, and Zhongning County in Ningxia is the geographical origin of the Goji berry. The fruit is typically orange-red (although there are black-purple varieties in Qinghai province), has a sweet taste, and matures from late June to late September. Goji berries have high sugar content and are extremely hygroscopic. In addition, they are easily damaged by worms. To eliminate pests, farmers apply pesticides during Goji growth period. Thus, Goji berries could contain pesticide residues.

${ }^{1}$ College of Pharmacy, Ningxia Medical University, Yinchuan 750004, China. ${ }^{2}$ College of Public Health and Management, Ningxia Medical University, Yinchuan 750004, China. ${ }^{3}$ College of Basic Medical Sciences, Ningxia Medical University, Yinchuan 750004, China. ${ }^{4}$ College of Resources and Environmental Science, Ningxia University, Yinchuan 750021, China. ${ }^{\circledR}$ email: jay70281@163.com 
In addition to pesticides, Goji berries absorb metals from cultivated soil. Further, some berries are fumigated to improve their appearance, and, thus, metals also introduced by this route. Consequently, pesticides and metals are the major hazardous chemicals in Goji berries. Previously, many types of pesticides and metals have been detected in Goji berries ${ }^{12,13}$. According to Chinese Pharmacopoeia $(2015)^{9}$, three pyrethroids and 12 organophosphates pesticides need to be detected in Chinese herbal medicine. However, three kinds of organophosphates pesticides are most frequently used in Goji berries by investigating the usage. Therefore, three pyrethroids pesticides (cypermethrin, fenvalerate, and deltamethrin) and three organophosphates pesticides (dichlorvos, omethoate, and malathion) were chosen in the current study. Also, according to Chinese Pharmacopoeia $(2015)^{14}$, five metals $(\mathrm{Pb}, \mathrm{Cd}, \mathrm{Cu} \mathrm{As}$, and $\mathrm{Hg}$ ) need to be detected in in Chinese herbal medicine. However, $\mathrm{Hg}$ was not detected in previous reports 8,15 , while $\mathrm{Ni}$ and $\mathrm{Zn}$, which would harmful to human health in high levels, were found in our pre-experiment ${ }^{16}$. Therefore, six metals $(\mathrm{Pb}, \mathrm{Cd}, \mathrm{Cu}, \mathrm{As}, \mathrm{Ni}$ and $\mathrm{Zn})$ were selected in the present study.

Numerous studies have focused on the biological activities of Goji berries, however, only a few studies have investigated pesticides and metals present Goji berries, especially with a focus on their associated health risks. Concerning pesticides, Li et al. ${ }^{12}$ identified 14 types of organophosphates pesticides in Goji berries using gas chromatography (GC). Huang et al. ${ }^{17}$ also used GC to simultaneously detect 50 kinds of organochlorine and pyrethroid pesticides in Goji berries. Chen et al. ${ }^{18}$ analyzed the etoxazole and pyridaben contents of Goji berries using GC method. Therefore, GC was also applied in the present study. These studies of pesticides in Goji berries did not discuss the associated health risks for consumers. While, recently, Fu et al. ${ }^{19}$ detected 8 pesticides and evaluated the associated dietary risk. Jing et al. ${ }^{20}$ analyzed 11 commonly used pesticide residues in Goji berries from Golmud area and conducted risk assessments for acute and chronic dietary exposures. Kim et al. ${ }^{21}$ monitored pesticides in Goji berries and assessed the short-term and highest long-term risks. However, the health risks in these studies were determined only through deterministic assessment. Considering the uncertainty of metal concentrations and the variability of exposure parameters, deterministic assessment may overestimate or underestimate risks ${ }^{22}$. Probabilistic assessment can solve this problem by providing probabilities and identifying priority chemicals for risk control. Therefore, both deterministic and probabilistic assessments were performed in the present work.

Several studies have reported the presence of metals in Goji berries. Sa et al. ${ }^{8}$ measured contents of macroand microelements, such as $\mathrm{Ni}$ and $\mathrm{Zn}$ in Goji berries using inductively coupled plasma-optical emission spectroscopy (ICP-OES). Kulaitienè et al. ${ }^{23}$ also used ICP-OES method to detect metals in Goji berries. Wojcieszek et al. ${ }^{13}$ used inductively coupled plasma mass spectrometry (ICP-MS) to quantify different metals in Goji berries. Rangsipanuratn et al. ${ }^{24}$ also used ICP-MS to detect heavy metals in Chinese medicinal herbs, including Goji berries. Fu et al. ${ }^{25}$ measured elements in some medicine food homologous (MFH) plants, including Goji berries, by ICP-tandem mass spectrometry (MS/MS). Xu et al. ${ }^{26}$ used different methods to detect five toxic metals in Goji berries. The $\mathrm{Pb}$ and $\mathrm{Cd}$ contents were detected by graphite furnace atomic absorption spectrometry (GFAAS), the $\mathrm{Cu}$ content was determined through FAAS, and the $\mathrm{Hg}$ and As contents were determined by atomic fluorescence spectrometry (AFS). ICP-OES combines a wide linear range, low detection limits, good sensitivity, widespread instrument availability, and reasonable $\operatorname{cost}^{8}$. Therefore, in this study, ICP-OES was used to identify and quantify the metals in Goji berries. In addition, most studies have focused on optimization methods or metal levels in different parts of Goji berries and the health risk of exposure through Goji berry consumption have been neglected. Therefore, an evaluation of the health risks posed by common metals in Goji berry is essential.

In summary, considering the simultaneous exposure to the main pollutants (pesticides and metals) in Goji berries and the uncertainty of risk assessment, this study was aimed to (1) analyze the concentrations of six pesticide residues (dichlorvos, omethoate, cypermethrin, fenvalerate, malathion, and deltamethrin) and six metals $(\mathrm{Pb}, \mathrm{Cd}, \mathrm{Cu}, \mathrm{As}, \mathrm{Ni}$ and $\mathrm{Zn})$ in Goji berries obtained from different sources in Ningxia, China; (2) determine the daily exposure to these hazardous chemicals from Goji berries; and (3) assess the health risks for consumers using deterministic and probabilistic methods.

\section{Results}

Levels of pesticides and metals in Goji berries. The methodological parameters for pesticides and metals were provided in Tables S1 and S2, respectively. All the parameters indicated that the methods were suitable for determining the contents of pesticides and metals.

The levels of pesticides and metals in Goji berries from different sources are summarized in Table 1. Five pesticides were detected in the Goji berry samples from plantations in a genuine production area. Meanwhile, only dichlorvos was detected in the supermarket samples. The levels of omethoate $(0.02 \pm 0.05 \mathrm{mg} / \mathrm{kg})$, dichlorvos $(0.02 \pm 0.03 \mathrm{mg} / \mathrm{kg})$, malathion $(0.02 \pm 0.03 \mathrm{mg} / \mathrm{kg})$, cypermethrin $(0.02 \pm 0.03 \mathrm{mg} / \mathrm{kg})$ and fenvalerate $(0.88 \pm 0.70 \mathrm{mg} / \mathrm{kg})$ were considerably below their MRLs of $0.5,1.0,1.0,0.5$ and $1.5 \mathrm{mg} / \mathrm{kg}^{27}$, respectively. Deltamethrin was not detected in any sample.

Except $\mathrm{Ni}$, the contents of other five metals in Goji samples from the plantations were higher than in case of those from the supermarket. The average Ni contents were $0.88 \pm 0.44 \mathrm{mg} / \mathrm{kg}$ in the plantation samples and $0.90 \pm 0.57 \mathrm{mg} / \mathrm{kg}$ in the supermarket samples, and there was no significant difference in Ni content between the two groups $(p=0.905,>0.05)$. However, there were significant differences in the $\mathrm{Cu}$ content $(p=0.031,<0.05)$ and very significant differences in the contents of $\mathrm{As}, \mathrm{Cd}, \mathrm{Pb}$, and $\mathrm{Zn}(p=0.000,<0.01)$. The MRL data of $\mathrm{Ni}^{28}$ and $\mathrm{Zn}^{29}$ are available for fruits but not for herbal medicines. The MRLs of Ni and $\mathrm{Zn}$ for fruits were applied to Goji berries because it is an MFH plant and consumed as a fruit. In such cases, the Ni and Zn levels were significantly higher than their MRLs. The average levels of $\mathrm{Cu}, \mathrm{Cd}, \mathrm{As}, \mathrm{Pb}$ were $8.70 \pm 2.70,0.10 \pm 0.07,0.20 \pm 0.23,0.35 \pm 0.27$ and $7.55 \pm 1.37,0.04 \pm 0.08,0.03 \pm 0.09,0.08 \pm 0.20 \mathrm{mg} / \mathrm{kg}$ in the plantation samples and supermarket samples, which were all lower than their MRL $(20 \mathrm{mg} / \mathrm{kg}, 0.3 \mathrm{mg} / \mathrm{kg}, 2 \mathrm{mg} / \mathrm{kg}, 5 \mathrm{mg} / \mathrm{kg})^{30}$. 


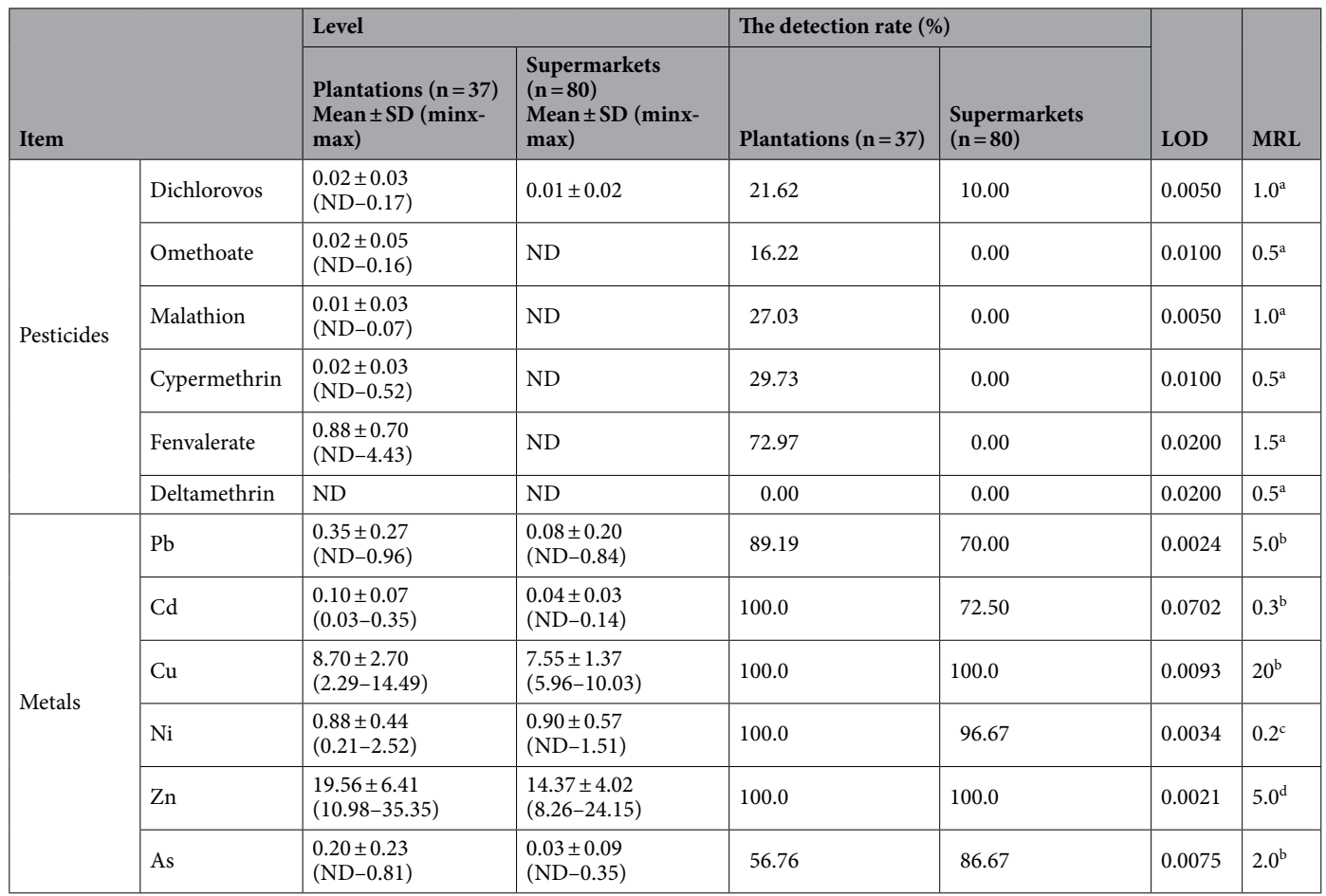

Table 1. Levels of pesticides and metals in Goji berries (mg/kg). $M R L$ means maximum residue limits, $N D$ means not detected. ${ }^{a}$ Means European maximum residue limits of pesticides ${ }^{27}$. ${ }^{b}$ Means China MRLs of metals for medicinal plants and preparations ${ }^{30}$. ${ }^{\mathrm{c}}$ Means China MRL of Ni for fruits ${ }^{28}$. ${ }^{\mathrm{d}}$ Means China MRL of $\mathrm{Zn}$ for fruits $^{29}$.

\begin{tabular}{|l|l|l|l|l|l|}
\hline Pesticides & Plantations & Supermarket & Metals & Plantations & Supermarket \\
\hline Dichlorovos & 0.0001 & 0.00006 & $\mathrm{~Pb}$ & 0.0030 & 0.0030 \\
\hline Omethoate & 0.0002 & - & $\mathrm{Cd}$ & 0.0009 & 0.0007 \\
\hline Malathion & 0.0002 & - & $\mathrm{Cu}$ & 0.0729 & 0.1213 \\
\hline Cypermethrin & 0.0001 & - & $\mathrm{Ni}$ & 0.0074 & 0.0076 \\
\hline Fenvalerate & 0.0074 & - & $\mathrm{Zn}$ & 0.1639 & 0.0637 \\
\hline Deltamethrin & - & - & $\mathrm{As}$ & 0.0017 & 0.0003 \\
\hline- & - & - & As-cancer & 0.00013 & 0.000019 \\
\hline
\end{tabular}

Table 2. Daily exposures to pesticides and metals in Goji berries ( $\mu \mathrm{g} /[\mathrm{kg}$ day $])$. - means not detected.

Exposure factors and daily exposures to pesticides and metals in Goji berries. The population diet survey result showed that the average daily amount of Goji berries consumed by humans is $1.37 \mathrm{~g}$ (DI/ daily intake), the EF (exposure frequency) is 145.7 days per year, ED (exposure duration) is 5.93 year, and the BW (body weight) of consumers is $64.81 \mathrm{~kg}$. Additional details are provided in Table S3. When Goji berries are used in tea or water, in this survey, the maximum dissolution rate was considered $30 \%{ }^{31}$. The daily exposures values for pesticides and metals in Goji berries were calculated using Eqs. (2) and (3), and the results are listed in Table 2 . The daily exposure values were used to calculate the health risks.

Human health risk assessment. Deterministic health assessment. Deterministic health risks were calculated using Eqs. (2) and (3), and the results are presented in Table 3. The HQ values of individual hazardous chemicals were all less than 1 , indicating the absence of a non-carcinogenic effect. For pesticides, the health risks of Goji berries from the plantations can be arranged as follows: fenvalerate > omethoate $>$ dichlorvos $>$ cypermethrin $>$ malathion. The orders of health risk from metals in Goji berries from plantations and supermarkets were $\mathrm{As}>\mathrm{Cu}>\mathrm{Cd}>\mathrm{Pb}>\mathrm{Zn}>\mathrm{Ni}$ and $\mathrm{Cu}>\mathrm{As}>\mathrm{Zn}>\mathrm{Ni}>\mathrm{Cd}>\mathrm{Pb}$, respectively.

The HI values were 0.0106 and 0.0037 for residents consuming Goji berries from plantations and supermarkets, respectively. The $\mathrm{HI}$ of pesticides $\left(\mathrm{HI}_{\mathrm{P}}\right)$ from the plantation samples was over 30 times higher than that of the supermarket samples. The $\mathrm{HI}$ of metals $\left(\mathrm{HI}_{\mathrm{M}}\right)$ from plantation samples was nearly 2.75 times than that of supermarket samples. For the Goji berries obtained from plantations, $\mathrm{HI}_{\mathrm{P}}$ accounts for $4.72 \%$ of $\mathrm{HI}_{\text {total }}$, and $\mathrm{HI}_{\mathrm{M}}$ 


\begin{tabular}{|l|l|l|l|l|l|}
\hline Pesticides & Plantations $(\mathbf{n}=\mathbf{3 7})$ & Supermarkets $(\mathbf{n}=\mathbf{8 0})$ & Metals & Plantations & Supermarkets \\
\hline Dichlorovos & $3.60 \times 10^{-5}$ & $1.51 \times 10^{-5}$ & $\mathrm{~Pb}$ & 0.0008 & 0.0002 \\
\hline Omethoate & $6.70 \times 10^{-5}$ & - & $\mathrm{Cd}$ & 0.0009 & 0.0003 \\
\hline Malathion & $5.03 \times 10^{-7}$ & - & $\mathrm{Cu}$ & 0.0018 & 0.0016 \\
\hline Cypermethrin & $7.25 \times 10^{-6}$ & - & $\mathrm{Ni}$ & 0.0004 & 0.0004 \\
\hline Fenvalerate & 0.0004 & - & $\mathrm{Zn}$ & 0.0005 & 0.0004 \\
\hline Deltamethrin & - & - & $\mathrm{As}$ & 0.0057 & 0.0008 \\
\hline $\mathrm{HI}_{\mathrm{P}}$ & 0.0005 & $1.51 \times 10^{-5}$ & $\mathrm{HI}$ & 0.0101 & 0.0037 \\
\hline $\mathrm{HI}_{\text {total }}$ of plantation & 0.0106 & & $\mathrm{HI}_{\text {total }}$ of supermarket & 0.0037 \\
\hline $\mathrm{R}_{\mathrm{As}}$ of plantations & $1.99 \times 10^{-7}$ & $\mathrm{R}_{\mathrm{As}}$ of supermarkets & $2.85 \times 10^{-8}$ \\
\hline
\end{tabular}

Table 3. Non-carcinogenic risk (hazard quotient (HQ) and hazard index (HI)) of pesticides and metals and carcinogenic risk $(\mathrm{R})$ of As in Goji berries. - means no available value. $\mathrm{HI}_{\mathrm{P}}$ means $\mathrm{HI}$ of pesticides and $\mathrm{HI}_{\mathrm{M}}$ means HI of metals.

\begin{tabular}{|c|c|c|c|c|c|}
\hline & Distribution & Parameters & $10 \%$ & $50 \%$ & $90 \%$ \\
\hline $\mathrm{HI}_{\mathrm{P}}$ of plantations & Lognormal & Location: 0.00 , mean: $5.80 \times 10^{-4}$, SD: $1.44 \times 10^{-3}$ & $2.82 \times 10^{-5}$ & $2.36 \times 10^{-4}$ & $1.29 \times 10^{-3}$ \\
\hline $\mathrm{HI}_{\mathrm{P}}$ of supermarkets & Lognormal & Location: 0.00 , mean: $7.00 \times 10^{-5}, \mathrm{SD}: 1.80 \times 10^{-4}$ & $9.30 \times 10^{-7}$ & $7.32 \times 10^{-6}$ & $3.99 \times 10^{-5}$ \\
\hline $\mathrm{HI}_{\mathrm{M}}$ of plantations & Lognormal & $\begin{array}{l}\text { Location: }-3.00 \times 10^{-5} \text {, mean: } 1.27 \times 10^{-2} \text {, SD: } \\
4.31 \times 10^{-2}\end{array}$ & $3.92 \times 10^{-4}$ & $3.83 \times 10^{-3}$ & $2.65 \times 10^{-2}$ \\
\hline $\mathrm{HI}_{\mathrm{M}}$ of supermarkets & Logic & Mean: $2.00 \times 10^{-3}$, scaling: $2.56 \times 10^{-3}$ & $4.49 \times 10^{-5}$ & $1.17 \times 10^{-3}$ & $8.42 \times 10^{-3}$ \\
\hline $\mathrm{R}_{\mathrm{As}}$ of plantations & Logic & Mean: 0.00 , scaling: 0.00 & $-5.80 \times 10^{-9}$ & $2.41 \times 10^{-8}$ & $4.96 \times 10^{-7}$ \\
\hline $\mathrm{R}_{\mathrm{As}}$ of supermarkets & Student T & Midpoint: 0.00 , scaling: 0.00 , freedom: 1 & $-4.13 \times 10^{-8}$ & $6.49 \times 10^{-10}$ & $6.56 \times 10^{-8}$ \\
\hline
\end{tabular}

Table 4. Statistics of the probabilistic estimation of the hazard index (HI) and R.

accounts for $95.28 \%$ of $\mathrm{HI}_{\text {total }}$. For the Goji berries obtained from supermarkets, nearly $100 \%$ of $\mathrm{HI}_{\text {total }}$ was $\mathrm{HI}_{\mathrm{M}}$. In summary, the Goji berries from plantations pose a greater risk than those obtained from supermarkets which can be related to the greater health risk posed by the metals than the pesticides.

The $\mathrm{R}$ values were $1.99 \times 10^{-7}$ and $2.85 \times 10^{-8}$ for customers taking Goji berries from plantations and supermarkets, respectively, which were lower than the negligible carcinogenic risks levels set by the USEPA $\left(10^{-6}\right)$. The result indicated that the carcinogenic risk posed by As of Goji berries in this study can be ignored.

Probabilistic health assessment. The best-fitting distributions of some EFs were simulated. For example, the metal contents in Goji berries from the plantations had beta, beta, normal, maximum extreme value, lognormal, and beta distributions for $\mathrm{Pb}, \mathrm{Cd}, \mathrm{Cu}, \mathrm{Ni}, \mathrm{Zn}$, and As, respectively (Table S4). Some EFs cannot simulate the bestfitting distributions because the data size does not meet the requirements. For example, dichlorvos was detected in Goji berries from the supermarkets in only eight samples. For these exposure factors, DI, EF, ED, and BW, the distributions had beta, geometry, lognormal, and beta distributions (Table S5; Fig. S1).

The results of the probabilistic estimation of health risks are summarized in Table 4. Firstly, the probabilistic results confirm the deterministic results. Most HI values were fitted to lognormal distributions (Fig. S2). The values of the 10th, 50th, and 90th $\mathrm{HI}_{\mathrm{P}}$ also suggest that the pesticides in Goji berries from plantations pose significantly higher health risks than those from the supermarkets. The 10th, 50th, and 90 th $\mathrm{HI}_{\mathrm{M}}$ values also indicate that the metals in Goji berries from the plantations pose a slightly higher health risk than those from the supermarkets. On the basis of the $\mathrm{HI}_{\text {total }}$ values, the Goji berries from plantations also pose a higher risk than those from supermarkets. Secondly, the probabilistic results indicate that a percentage of the population will be exposed to levels exceeding the standards. The results in Table 4 show that no population exceeded 1, indicating that the consumption Goji berries is safe.

In the probabilistic estimation of As carcinogenic risk of Goji berries from plantations, the 50th and 90th percentile R values were $2.41 \times 10^{-8}$ and $4.96 \times 10^{-7}$, which were less than $10^{-6}$. For Goji berries from supermarkets, the 50th and 90th percentile R values were $6.49 \times 10^{-9}$ and $6.56 \times 10^{-8}$, which were also lower than $10^{-6}$. In terms of the $\mathrm{R}$ values, the Goji berries from plantations pose a higher risk than those from supermarkets.

Sensitivity results. The most sensitive factors were calculated (Fig. S3). For the total non-carcinogenic risk of Goji berries from plantations, the most sensitive factors were exposure frequency (55.3\%), daily intake (27.4\%) of Goji berries and As content (17.4\%). Concerning the total risk of Goji berries from supermarkets, the most sensitive factors were exposure frequency $(48.3 \%)$, daily intake $(25.3 \%)$ of Goji berries and the contents of $\mathrm{As}(24.7 \%), \mathrm{Cd}(0.9 \%)$ and $\mathrm{Pb}(0.7 \%)$. In summary, exposure frequency, daily intake and As exposure were the most sensitive factors that influencing the health risk results, and control the frequency of intake is the most important way to reduce the non-carcinogenic risk of Goji berries.

For the carcinogenic risk of Goji berries from plantations, the most sensitive factors were As content (68.9\%), exposure frequency (14.6\%), exposure duration (8.9\%), and daily intake (7.3\%). For the carcinogenic risk of 


\begin{tabular}{|c|c|c|c|}
\hline Medicine food homologous plants & Non-carcinogenic risk (HI) & Carcinogenic risk (R) & References \\
\hline \multirow{2}{*}{ Lycium barbarum $\mathrm{L}$. } & 0.0106 (plantations) & $1.99 \times 10^{-7}$ (plantations) & \multirow{2}{*}{ The present study } \\
\hline & 0.0037 (supermarkets) & $2.85 \times 10^{-8}$ (supermarkets) & \\
\hline Panax notoginseng & 0.013 & $2.1 \times 10^{-6}$ & Zhu et al. ${ }^{40}$ \\
\hline Glycyrrhizae radix & 0.042 & $4.11 \times 10^{-6}$ & Zhu et al. ${ }^{41}$ \\
\hline Astragalus membranaceus & 0.049 & $4.3 \times 10^{-6}$ & Tian et al. ${ }^{42}$ \\
\hline Prunella vulgaris & 0.029 & - & Cao and $\mathrm{Zhu}^{43}$ \\
\hline Yam & - & $5.9 \times 10^{-5}$ & Zhu et al. ${ }^{44}$ \\
\hline Haw & - & $2.4 \times 10^{-5}$ & Zhu et al. ${ }^{44}$ \\
\hline Jujube & - & $8.3 \times 10^{-7}$ & Zhu et al. ${ }^{44}$ \\
\hline
\end{tabular}

Table 5. The non-carcinogenic health risk and carcinogenic risk of metals in medicine food homologous plants. - means no available data.

Goji berries from supermarkets, the most sensitive factors were As content (98.5\%), exposure frequency (0.6\%), exposure duration $(0.5 \%)$, and daily intake $(0.4 \%)$. Therefore, controlling As content is the most effective way to reduce the carcinogenic risk of Goji berries.

\section{Discussion}

Levels of pesticides and metals in Goji berries. Huang et al. ${ }^{17}$ found that cypermethrin and fenvalerate contents were $0.018-0.059$ and $0.016-0.090 \mathrm{mg} / \mathrm{kg}$, respectively, which are both remarkably lower than those in the current study. The pesticide levels were determined on the basis of many factors, such as the time and frequency of pesticide spraying and the types of pesticides. Pesticides were not detected in most supermarket samples. This result could arise for a variety of reasons. For example, some plantation samples may spray pesticides before we collect samples. Furthermore, after sale, many techniques are used to remove pesticides. Zhang et al. ${ }^{32}$ found that sun-drying and oven-drying can reduce pesticide residues by $10-70 \%$ and the removal rate increased as the temperature increased. The drying temperature in the present study $\left(60^{\circ} \mathrm{C}\right)$ was higher than that in their study $\left(45^{\circ} \mathrm{C}\right.$ to $50^{\circ} \mathrm{C}$ ), so it could be speculated that the removal rate of pesticides in the present study was a litter higher. Ye et al. ${ }^{33}$ used ozone and alkali liquor to degrade four organophosphates pesticides and achieved a dichlorvos removal of $97.83 \%$. Zhou et al. ${ }^{34}$ used a gas-phase surface discharge plasma method and verified that a large proportion of omethoate and dichlorvos (>95\%) could be removed from Goji berries. This may explain why pesticides in Goji samples from the supermarket were nearly undetectable. Goji berries are always consumed as dry berries and washed before they are eaten. In order to close the real situation, samples were washed and dried in the current study. And according to the previous reports, the pesticide residues are bound to washed off or breakdown. The exact rate of pesticides and metals washed off or breakdown should be conducted in further study.

Kai et al. ${ }^{35}$ collected 10 Goji samples from a planting base in Zhongning, Ningxia, and found metal contents were $0.13 \mathrm{mg} / \mathrm{kg}(\mathrm{Pb}), 0.07 \mathrm{mg} / \mathrm{kg}(\mathrm{Cd}), 6.00 \mathrm{mg} / \mathrm{kg}(\mathrm{Cu}), 0.51 \mathrm{mg} / \mathrm{kg}(\mathrm{Ni}), 16.80 \mathrm{mg} / \mathrm{kg}(\mathrm{Zn})$, and $0.01 \mathrm{mg} / \mathrm{kg}$ (As), respectively. Our results were $2.7(\mathrm{~Pb}), 0.70(\mathrm{Cd}), 1.45(\mathrm{Cu}), 0.58(\mathrm{Ni})$, and $20(\mathrm{As})$ times higher than their results. Possible reason of the significant difference of As level was the samples were only from one planting base in their study. Wang et al. ${ }^{36}$ measured the heavy metals of Goji berries from peasant household in Yinchuan, Ningxia, and the contents of 4 heavy metals were $0.11 \mathrm{mg} / \mathrm{kg}(\mathrm{Pb}), 0.029 \mathrm{mg} / \mathrm{kg}(\mathrm{Cd}), 1.41 \mathrm{mg} / \mathrm{kg}(\mathrm{Ni})$, and $0.20 \mathrm{mg} / \mathrm{kg}$ (As), respectively. Their results were similar with ours. However, there are few researches on heavy metals of Goji berries from supermarkets. Some techniques, such as supercritical fluid extraction and flocculation, are used to remove metals in herbal medicines ${ }^{37}$. This may clarify why metals in Goji samples from the supermarket were relatively low. Furthermore, the contents of metals are varied from different areas of the world. For example, $\mathrm{Zn}, \mathrm{Cu}, \mathrm{Pb}$, and $\mathrm{Cd}$ in Goji berries from Lithuania ${ }^{23}, \mathrm{Cu}$ and $\mathrm{Zn}$ levels in Goji berries from Sante (Poland) ${ }^{24}, \mathrm{Ni}, \mathrm{Zn}$ and $\mathrm{Pb}$ levels in Goji berries from Turkey ${ }^{38}$, were all lower than that of our results, while As, $\mathrm{Cd}$, and $\mathrm{Pb}$ in most Goji samples were higher than that of our results ${ }^{25}$. Even in the nearby areas, the results can be different. Liu et al. ${ }^{39}$ reported metals varied in Goji berries from different areas of Ningxia. Thus, further study of the correlation between metal contents and cultivation region is required.

Health risk assessment. The levels of pesticides in Goji berries pose a low risk after chronic exposure in this study. Similar health risks arising from pesticides in Goji berries have been reported previously. Fu et al. ${ }^{19}$ found that pesticides in Goji berries do not result in health risks because the HQ is considerably less than 1. Jing et al. ${ }^{20}$ calculated the acute and chronic dietary exposure risks of pesticides through Goji berry consumption and found the risk to be low. Kim et al. ${ }^{21}$ determined the threat of pesticides in Goji berries to consumers at shortterm and highest long-term exposure and no significant health risk was detected. Hence, the levels of pesticides in Goji berries do not pose obvious health risk to consumers after chronic exposure.

Concerning the health risk assessment of metals, no other investigations on Goji berries are available. We previously studied other MFH plants and found that the presence of metals in these types of plants does not pose non-carcinogenic health risks (Table 5). For example, the HI values of metals in Panax notoginseng ${ }^{40}$, Glycyrrhizae radix ${ }^{41}$, Astragalus membranaceus ${ }^{42}$, and Prunella vulgaris ${ }^{43}$ were $0.013,0.042,0.049$, and 0.029 , respectively, which are considerably less than 1 . 
The result indicated that the carcinogenic risk posed by As of Goji berries in this study can be ignored. However, the carcinogenic risk posed by As is always higher than the negligible carcinogenic risk level $\left(10^{-6}\right)$ but lower than the maximum acceptable carcinogenic risk level $\left(10^{-4}\right)$ (Table 5). For example, the $R$ values of As in Panax notoginseng ${ }^{40}$, Glycyrrhizae radix ${ }^{41}$, Astragalus membranaceus ${ }^{43}$, yam, and haw ${ }^{44}$ are $2.1 \times 10^{-6}, 4.11 \times 10^{-6}$, $4.3 \times 10^{-6}, 5.9 \times 10^{-5}$, and $2.4 \times 10^{-5}$, respectively. The carcinogenic risk value of As in jujube ${ }^{44}$ is $8.3 \times 10^{-7}$, which is far less than the negligible level (Table 5).

Goji berries are mostly taken as an herbal medicine, the ADI of hazardous chemicals from medicines (ADImedicine) should not exceed $1 \%$ of ADI, as suggested by the $\mathrm{WHO}^{45}$, and the factors of DI, EF, ED are also totally different. Assuming this condition, the exposure values, HQ of individual hazardous chemicals, and HI of combined hazardous chemicals are different from the present values which should be verified in further study. In addition to Goji berries, many other foods, such as rice, vegetables, and fruits, expose residents to pesticides and metals ${ }^{46-48}$. Considering daily dietary intake, total health risk should not be disregarded. Moreover, ingestion methods, such as direct consumption, dissolution in water, and use as medicine, will seriously affect the results. Thus, these factors should be the focus of future studies.

In summary, it's more accurate to evaluate health risk caused by main chemical contaminates (pesticides and metals) in Goji berry simultaneously and assess health risk by probabilistic methods considering the uncertainty. Furthermore, the related ways of controlling risk are provided. However, there are still some limitations, the removal rate of pesticides and metals by washing and drying, the correlations between metals and regions, the health risk considering more factors should be investigated in further study.

\section{Methods}

Sample collection and laboratory pretreatment. Goji samples (approximately $500 \mathrm{~g}$ when fresh) were collected from 37 plantations in Zhongning County (genuine production area) in Ningxia Province, China in June 2020, as shown in Fig. 1. All the Goji berries were cultivated by local famers. In each plantation, five subsamples were collected. The Goji samples were packed into bags, marked, immediately transported to the laboratory, and stored at $4{ }^{\circ} \mathrm{C} .80$ commercial Goji samples were purchased from local 15 supermarkets in Yinchuan, Ningxia, China. 5-6 samples at different prices were obtained in each supermarket. All Goji samples were identified by Professor Liming Zhang, School of Pharmacy, Ningxia Medical University.

In order to better simulate the actual situation of Goji berries intake, the Goji samples were first washed with tap water and, then, with deionized water. Subsequently, they were dried in an oven (DHG-9030A, China) at $60{ }^{\circ} \mathrm{C}$ for approximately 5 days (the moisture was less than 13\%). Finally, the dried samples were ground using high-speed universal pulverizer (FW100, China), sifted through a 60-mesh sieve, and stored in sealed sample bags until analysis.

Pesticide determination. Pesticide determination was carried out with reference to the 2015 edition of China Pharmacopoeia $a^{9}$, "Determination of pesticide residues." Three pyrethroids (cypermethrin, fenvalerate, and deltamethrin) and three organophosphates (dichlorvos, omethoate, and malathion) pesticides were detected by gas chromatograph (GC) (Agilent 7890B, USA) method. The chromatographic conditions for GC analysis for pesticides detection are summarized in Table S5. Three replicates were performed to ensure precision. Standard curves were drawn using data obtained from a series of standard solutions. A blank control, as well as recovery test, was used to ensure accuracy. The limits of detection (LOD) and limits of quantification (LOQ) of each hazardous chemical were calculated with signal-to-noise (S:N) ratios of 3:1 and 10:1, respectively.

Metal determination. The determination of metals in Goji berries has been reported previously ${ }^{16}$. First, $0.2 \mathrm{~g}$ dried Goji berry samples were digested using a mixture of $\mathrm{HNO}_{3}$ and $\mathrm{HClO}_{4}(4: 1, V: V)$ in low temperature combined digester (ED54-iTouch, China), and the six metals were quantified via ICP-OES (Varian 710-ES, USA). Three replicates were performed to ensure precision. Standard curves were drawn using a series of standard solutions. A blank control, a recovery test, and a standard reference were employed to ensure accuracy. The LOD and LOQ were also calculated. The conditions for the analysis of metals are provided in Table S7.

Population diet survey. Determination of sample size. According to the principle of random sampling, representative objects of edible Goji berries from a producing area were selected; the sample size was calculated according to Eq. (1):

$$
\mathrm{n}=\frac{u^{2} \times P(1-P)}{E^{2}}
$$

where $\mathrm{n}$ is the sample number, $u$ is the critical value corresponding to a certain degree of confidence, $P$ is the dispersion degree of the sample, and $E$ is the sampling error range. The confidence level was set to $95 \%$, so $u$ was 1.96 , and $E$ was $\pm 5 \%$. The sample number was the largest when $P$ was 0.5 , and the calculated sample size was 384 .

Questionnaire on Goji consumption. To determine the Goji berry consumption habits of consumers, questionnaire was conducted in the cities of Zhongning and Yinchuan in Ningxia, China in June 2020. A total of 712 inhabitants, were asked to join our survey and 558 respondents participated in our face-to-face interview or online questionnaire eventually with the response rate of $78.37 \%$. According to expert's recommendation, the participants who consumed Goji berries at least once a week and last for three months or more were taken into account of chronic exposure. They were asked to recall the quantity, frequency, duration, and way of their con- 


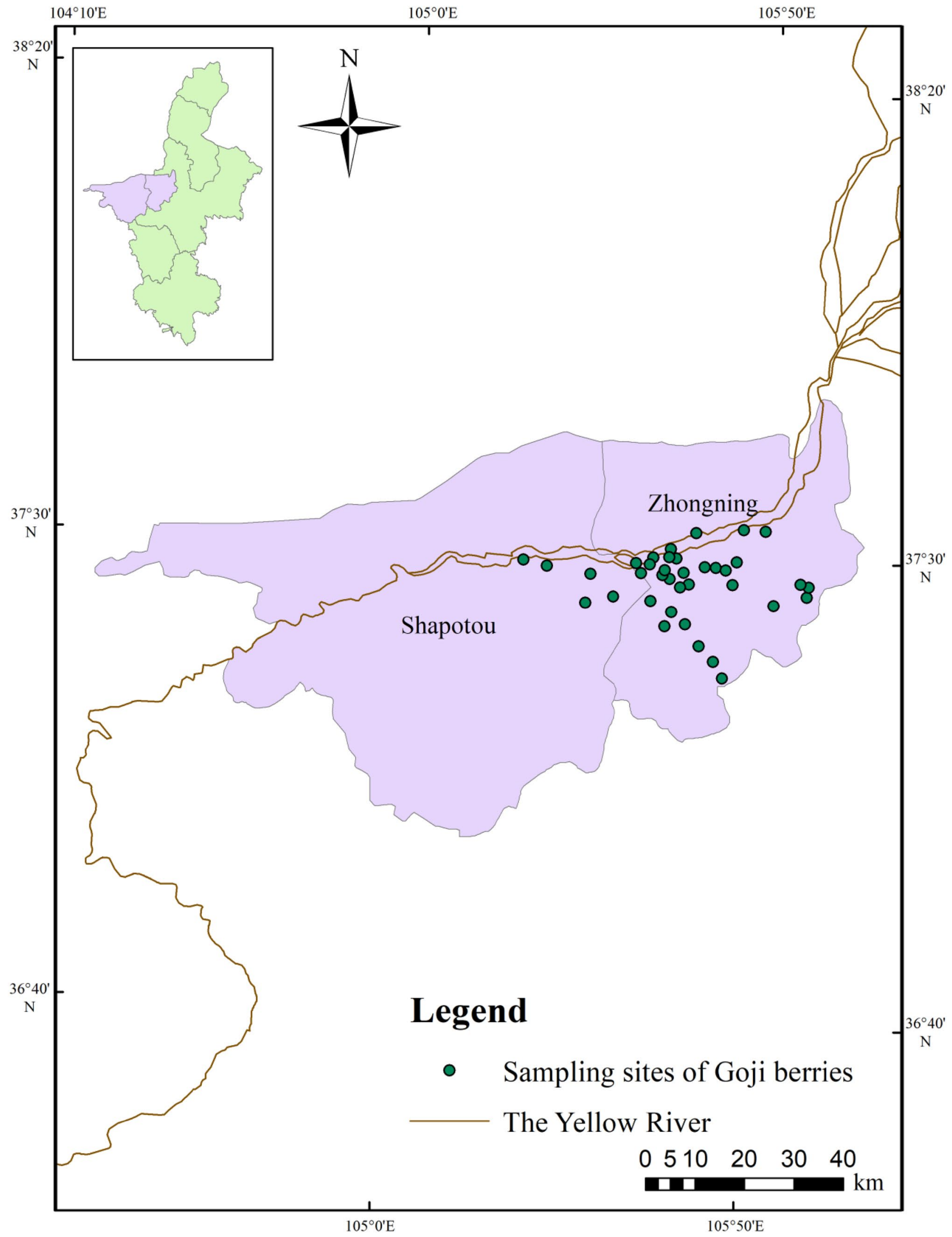

Figure 1. Sampling sites of Goji berries from plantations in genuine producing area (represents sampling sites) (data source: http://bbs.3s001.com/forum.php?mod=viewthread\&tid=133741\&page=1\#pid2930761). 


\begin{tabular}{|l|l|l|l|}
\hline Pesticides & ADI $(\mu \mathrm{g} /[\mathbf{k g}$ day $])$ & Metals & ADI $(\mu \mathrm{g} /[\mathrm{kg}$ day $])$ \\
\hline Dichlorovos & 4 & $\mathrm{~Pb}$ & 3.6 \\
\hline Omethoate & 3 & $\mathrm{Cd}$ & 1 \\
\hline Malathion & 300 & $\mathrm{Cu}$ & 40 \\
\hline Cypermethrin & 20 & $\mathrm{Ni}$ & 20 \\
\hline Fenvalerate & 20 & $\mathrm{Zn}$ & 300 \\
\hline Deltamethrin & 10 & $\mathrm{As}$ & 0.3 \\
\hline
\end{tabular}

Table 6. Allowable daily intake (ADI) of pesticides and metals ( $\mu \mathrm{g} /[\mathrm{kg}$ day $])$. ADI of pesticides ${ }^{51}$. ADI of metals $^{52}$.

sumption of Goji berry, as well as to provide their age and body weight. 401 valid questionnaires were collected. The Goji berry consumption information was used for health risk assessment.

Daily exposure. Goji berries are typically consumed by adults as a health product. The daily exposure to pesticides and metals through Goji berry consumption was calculated using Eqs. (2) and (3) (US Environmental Protection Agency) ${ }^{49}$ :

$$
\begin{gathered}
E X P O=\frac{C \times D I \times E F \times E D}{B W \times A T}, \\
E X P O_{A s}=\frac{c \times D I \times E F \times E D}{B W \times L T},
\end{gathered}
$$

Equation (2) was used to calculate non-carcinogenic exposure and Eq. (3) was to calculate As carcinogenic exposure. Here, EXPO $(\mu \mathrm{g} /[\mathrm{kg}$ day $])$ represents the exposure to hazardous chemicals, $\mathrm{C}(\mathrm{mg} / \mathrm{kg})$ is the concentration of hazardous chemicals in Goji berries, DI (g/day) is the daily intake of Goji berries, EF (day/year) is the exposure frequency, ED (year) represents the exposure duration, BW (kg) is the body weight of residents, AT (year) is the average exposure time $(\mathrm{ED} \times 365$ day/year), LT (year) is the average lifespan of the consumers and is $76.34^{50}$.

Health risk assessment. Deterministic assessment. All six pesticides exhibit non-carcinogenic effects through chronic oral exposure. $\mathrm{Cd}, \mathrm{Pb}, \mathrm{Cu}, \mathrm{Ni}$, and $\mathrm{Zn}$ pose non-carcinogenic health risks, and As poses noncarcinogenic and carcinogenic health risks. Non-carcinogenic individual hazardous chemical levels can be assessed by estimating the hazard quotient (HQ) value, which can be calculated using Eq. $(4)^{49}$ :

$$
H Q=E X P O / A D I,
$$

here, EXPO ( $\mu \mathrm{g} /[\mathrm{kg}$ day]) represents the exposure to hazardous chemicals, and ADI ( $\mu \mathrm{g} /[\mathrm{kg}$ day]) is the allowable daily intake. The values of each hazardous chemical are summarized in Table 6 . The ADI values for six pesticides were set by the World Health Organization $(\mathrm{WHO})^{51}$, and the ADI values for six metals were suggested by the USEPA ${ }^{52}$. The ADIs of the pesticides were 4, 3, 300,20,20, and $10 \mu \mathrm{g} /[\mathrm{kg}$ day] for dichlorvos, omethoate, malathion, cypermethrin, fenvalerate, and deltamethrin, respectively. The ADIs of $\mathrm{Pb}, \mathrm{Cd}, \mathrm{Cu}, \mathrm{Ni}, \mathrm{Zn}$, and As were $3.6,1,40,20,300$, and $0.3 \mu \mathrm{g} /[\mathrm{kg}$ day], respectively. When the interactions between hazardous chemicals are not considered, the hazard index (HI; Eq. 5) can be calculated to evaluate the total non-carcinogenic health risk upon exposure to more than one hazardous chemical ${ }^{53}$,

$$
H I=\sum_{1}^{n} H Q_{n} .
$$

The carcinogenic risk $(R)$ posed by As was evaluated using Eq. $(6)^{54}$,

$$
R=S F \times E X P O,
$$

here, $\mathrm{SF}$ is the slope factor with a suggested value of $1.5 \mathrm{mg} /[\mathrm{kg} \text { day }]^{55}$.

Probabilistic assessment. Average values are used in deterministic assessment; but they cannot reflect the uncertainty and variability in health risk evaluation. Consequently, probabilistic assessment was used to assess the probabilistic distribution of daily exposure to hazardous chemicals and harmful risks. Monte Carlo simulation is widely used in probabilistic estimation ${ }^{56}$ and can be described as follows. First, the best-fitting distributions of exposure parameters are obtained by fitting several parametric distributions (e.g., lognormal, gamma, and beta) and selecting the distribution with the best statistical results, such as the Anderson-Darling test and chi-square $\left(\chi^{2}\right)$ test. Then, exposure parameter values are randomly selected from the best distribution and simulated with thousands of iterations to obtain stable distributions of exposures and health risks. The results of probabilistic estimations are frequently presented in distribution, distribution parameters, and percentile values (e.g., 10th, 50th, and 90th). The entire process was carried out using Crystal Ball. 
Statistical methods. Mean and standard deviation (SD) were calculated in Microsoft Excel 2010 (Microsoft Ins., USA). The Mann-Whitney U test was conducted in SPSS 21.0 (IBM Ins., USA). The determination of the best-fitting distribution and the Monte Carlo simulation were performed using Crystal Ball software (Oracle@ Ins., USA). ArcGIS Desktop 10 (ESRI Ins., USA, Authorization number: EFL564098460) was used to map the sampling sites.

Ethics approval and consent to participate. The collection of Goji samples from plantations was permitted by local famers orally. The plant collection and the study complied with local (Ningxia) and national (China) regulations. The study was approved by the institutional research ethics committee of Ningxia Medical University, and written informed consent was obtained from each participant. All the methods in this manuscript were carried out in accordance with relevant guidelines and regulations.

\section{Data availability}

The datasets used and/or analyzed during the current study available from the corresponding author on reasonable request.

Received: 15 September 2021; Accepted: 14 December 2021

Published online: 12 January 2022

\section{References}

1. Potterat, O. Goji (Lycium barbarum and L. chinense): Phytochemistry, pharmacology and safety in the perspective of traditional uses and recent popularity. Planta Med. 76(01), 7-19 (2010).

2. Amagase, H. \& Farnsworth, N. A review of botanical characteristics, phytochemistry, clinical relevance in efficacy and safety of Lycium barbarum fruit (Goji). Food Res. Int. 44(7), 1702-1717 (2011).

3. Kulczyński, B. \& Gramza-Michałowska, A. Goji berry (Lycium barbarum): Composition and health effects-a review. Pol. J. Food Nutr. Sci. 66(2), 67-76 (2016).

4. Benchennouf, A., Grigorakis, S., Loupassaki, S. \& Kokkalou, E. Phytochemical analysis and antioxidant activity of Lycium barbarum (Goji) cultivated in Greece. Pharm. Biol. 55(1), 596-602 (2017).

5. Wawruszak, A., Czerwonka, A., Okła, K. \& Rzeski, W. Anticancer effect of ethanol Lycium barbarum (Goji berry) extract on human breast cancer T47D cell line. Nat. Prod. Res. 30(17), 1993-1996 (2016).

6. Sultan, A. T. M., Al-Salhie, K. C. K. \& Shawket, T. F. Effect of adding Lycium barbarum L. extract to drinking water on some productive traits of Japanese quail (Coturnix japonica). Basrah J. Agric. Sci. 32(2), 208-212 (2019).

7. Sharwani, A. A., Narayanan, K. B., Khan, M. E. \& Han, S. S. Sustainable fabrication of silver-titania nanocomposites using goji berry (Lycium barbarum L.) fruit extract and their photocatalytic and antibacterial applications. Arab. J. Chem. 14(12), 103456 (2021).

8. Sá, R. R. et al. Multielementar/centesimal composition and determination of bioactive phenolics in dried fruits and capsules containing Goji berries (Lycium barbarum L). Food Chem. 273, 15-23 (2019).

9. Cariddi, C., Mincuzzi, A., Schena, L., Ippolito, A. \& Sanzani, S. M. First report of collar and root rot caused by Phytophthora nicotianae on Lycium barbarum. J. Plant Pathol. 100(2), 361-361 (2018).

10. Skenderidis, P. et al. Chemical properties, fatty-acid composition, and antioxidant activity of goji berry (Lycium barbarum L. and Lycium chinense Mill.) fruits. Antioxidants. 8(3), 60 (2019).

11. Olech, M. et al. Polyphenol composition and antioxidant potential of instant gruels enriched with Lycium barbarum L. Fruit. Mol. 25, 4538 (2020).

12. Li, L. et al. Determination of organophosphorus pesticides in Lycium barbarum by gas chromatography with flame photometric detection. J. AOAC Int. 90(1), 271-276 (2007).

13. Wojcieszek, J., Kwiatkowski, P. \& Ruzik, L. Speciation analysis and bioaccessibility evaluation of trace elements in goji berries (Lycium barbarum, L.). J. Chromatogr. A 1492, 70-78 (2017).

14. Chinese Pharmacopoeia Commission. Chinese Pharmacopoeia (2015) (in Chinese).

15. Gogoasa, I. et al. Goji berries (Lycium barbarum) as a source of trace elements in human nutrition. J. Agroaliment. Process. Technol. 20(4), 369-372 (2014).

16. Zhang, Y. et al. Content comparison of seven harmful elements in Lycium barbarum berry from the geographical origin fields and supermarkets. Int. J. Agric. Biol. 24, 1565-1572 (2020).

17. Huang, X., Xue, J., Wang, Y., Wu, X. \& Tong, H. Rapid simultaneous determination of organochlorine and pyrethroid pesticide residues in Lycium barbarum L. using gas chromatography with electron-capture detector. Anal. Methods. 4(4), 1132-1141 (2012).

18. Chen, H. et al. Residue, dissipation, and safety evaluation of etoxazole and pyridaben in Goji berry under open-field conditions in the China’s Qinghai-Tibet Plateau. Environ. Monit. Assess. 191(8), 517 (2019).

19. Fu, Y. et al. Determination of eight pesticides in Lycium barbarum by LC-MS/MS and dietary risk assessment. Food Chem. 218, 192-198 (2017).

20. Jing, X., Qi, J. \& Yang, H. Risk assessment of pesticide residues in wolfberry from Golmud area. Ecol. Environ. Sci. 28(5), 1007-1012 (2019).

21. Kim, J., Shin, J., Park, C. G. \& Lee, S. H. Pesticide residue monitoring and risk assessment in the herbal fruits Schisandra chinensis, Lycium chinense, and Cornus officinalis in Korea. Food Sci. Biotechnol. 30(1), 137-147 (2021).

22. Yang, S. et al. Status assessment and probabilistic health risk modeling of metals accumulation in agriculture soils across China: A synthesis. Environ Int. 128, 165-174 (2019).

23. Kulaitiené, J. et al. Concentrations of minerals, soluble solids, vitamin C, carotenoids and toxigenic elements in organic goji berries (Lycium barbarum L.) cultivated in Lithuania. Biol. Agric. Hortic. 36(2), 130-140 (2020).

24. Rangsipanuratn, W. et al. Detection of microbes, aflatoxin and toxic heavy metals in Chinese medicinal herbs commonly consumed in Thailand. Pharm. Sci. Asia. 44(3), 162-171 (2017).

25. Fu, L., Shi, S. \& Chen, X. Accurate quantification of toxic elements in medicine food homologous plants using ICP-MS/MS. Food Chem. 245, 692-697 (2018).

26. Xu, X., Hu, S. \& Pan, R. Determination of heavy metals and organochlorine pesticides in Lycium barbarum. Chin. J. Health Lab. Tec. 24, 1487-1492 (2014) (in Chinese).

27. European Pharmacopoeia Commission. European pharmacopoeia (2014).

28. Fu, Y., Hu, X. \& Yu, S. Study on the tolerance limit of nickel in foods. J. Zhejiang Acad. Med. Sci. 37, 9-11 (1999).

29. Chinese Ministry of Health. Tolerance limit of Zn in Foods. GB 13106-1991 (1991) (in Chinese). 
30. Ministry of Foreign Trade and Economic Cooperation of Chsina. Greentrade standards of importing \& exporting medicinal plants \& preparations. WM/T2-2004 (2001) (in Chinese).

31. Luo, Y., Huang, W., Long, Z. \& Deng, M. Contents and dissolutions of 8 heavy metals in before and after decoctions of traditional Chinese medicines by ICP-MS. Chin. J. Spectrosc. Lab. 29(02), 925-928 (2012).

32. Zhang, Y., Wu, Y. \& Niu, Y. Effects of drying methods on pesticide residues in wolfberry and its dietary exposure assessment. Food Res. Dev. 37(13), 176-180 (2016).

33. Ye, Y., Suo, Y., Han, L., Hu, N. \& Wang, H. Degradation of four kinds of organophosphorus pesticides in Lycium barbarum L. by using ozone and alkali liquor respectively. Sci. Tech. Food Ind. 35(04), 101-104 (2014).

34. Zhou, R. et al. Removal of organophosphorus pesticide residues from Lycium barbarum by gas phase surface discharge plasma. Chem. Eng. J. 342, 401-409 (2018).

35. Kai, J. R., Li, C. H., Zhao, D. Q. \& Wang, C. Y. Element chemical analysis of difference region and cultivar Lycium barbarum in Ningxia. Food Ferment. Ind. 46, 257-264 (2020) (in Chinese).

36. Wang, C. Y. \& Zhang, Y. Determination of 12 kinds of heavy metal in Lycium by ICP-MS. Food Res. Dev. 33(11), 148-150 (2012) (in Chinese).

37. Cheng, Y. et al. Research on application of heavy metal removal in Traditional Chinese Medicine. J. Anhui Agric. Sci. 39(9), 5340-5342 (2011) (in Chinese).

38. Endes, Z. et al. Physico-chemical properties, fatty acid composition and mineral contents of goji berry (Lycium barbarum L.) fruit. J. Agroaliment. Process Technol. 21(1), 36-40 (2015).

39. Liu, Y. et al. Survey on the contents of the trace elements in medlar from different areas. J. Insp. Quar. 22(3), 32-35 (2012) (in Chinese).

40. Zhu, M. et al. Determination of the heavy metal levels in Panax notoginseng and the implications for human health risk assessment. Hum. Ecol. Risk Assess. 21(5), 1218-1229 (2015).

41. Zhu, M., Zhu, X. \& Feng, N. Evaluation of health risks and determination of limited concentrations of heavy metals in Glycyrrhizae Radix et Rhizoma. Chin. Tradit. Pat. Med. 38(8), 1771-1776 (2016) (in Chinese).

42. Tian, R. et al. Health risk of five common heavy metals exposure based on Astragalus membranaceus ingestion. J. Mod. Med. Health. 21, 3287-3289 (2018) (in Chinese).

43. Cao, J. \& Zhu, M. Non-carcinogenic risk of heavy metal exposure based on Traditional Chinese Medicine Prunella vulgaris $\mathrm{L}$. ingestion. J. Energy Res. Manag. 3, 53-55 (2016) (in Chinese).

44. Zhu, M., Feng, N., Yan, Q. \& Zhu, X. Carcinogenic risk assessment of arsenic in three medicine-food Chinese traditional medicine. Shizhen Guo Yi Guo Yao. 29(11), 2601-2603 (2018) (in Chinese).

45. World Health Organization. Quality control methods for medicinal plant materials-Revised draft update (2005).

46. Naseri, M., Vazirzadeh, A., Kazemi, R. \& Zaheri, F. Concentration of some heavy metals in rice types available in Shiraz market and human health risk assessment. Food Chem. 175, 243-248 (2015).

47. Bempah, C., Donkor, A., Yeboah, P., Dubey, B. \& Osei-Fosu, P. A preliminary assessment of consumer's exposure to organochlorine pesticides in fruits and vegetables and the potential health risk in Accra Metropolis, Ghana. Food Chem. 128(4), 1058-1065 (2011).

48. Bhanti, M. \& Taneja, A. Contamination of vegetables of different seasons with organophosphorous pesticides and related health risk assessment in northern India. Chemosphere 69(1), 63-68 (2007).

49. USEPA. Guidelines for Exposure Assessment. EPA/600/Z-92/001. https://www.epa.gov/risk/guidelines-exposure-assessment. Accessed 28 Dec 2020 (U.S. Environmental Protection Agency, Risk Assessment Forum, 1992).

50. National Bureau of Statistics. China Statistical Yearbook. http://www.stats.gov.cn/tjsj/ndsj/2019/indexch.htm. Accessed 28 Dec 2020 (2019).

51. WHO. Inventory of evaluations performed by the Joint Meeting on Pesticide Residues (JMPR). http://apps.who.int/pesticide-resid ues-jmpr-database. Accessed 28 Dec 2020.

52. USEPA. Risk-based concentration table (United States Environmental, Protection Agency, 2000).

53. USEPA. Guidance for Performing Aggregate Exposure and Risk Assessments (U.S. Environmental Protection Agency, Office of Pesticide Programs, 1999).

54. USEPA. Guidelines for Carcinogen Risk Assessment, NCEA-F-0644. EPA/630/P-03/001F. https://www.epa.gov/risk/guidelinescarcinogen-risk-assessment. Accessed 28 Dec 2020 (U.S. Environmental Protection Agency, Risk Assessment Forum, 2005).

55. USEPA. Integrated Risk Information System (IRIS). http://www.epa.gov/iris/subst/0278.htm. Accessed 28 Dec 2020 (2010).

56. Bourne, S. et al. A Monte Carlo method for probabilistic hazard assessment of induced seismicity due to conventional natural gas productiona Monte Carlo method for probabilistic hazard assessment of induced seismicity. Bull. Seismol. Soc. Am. 105(3), $1721-1738$ (2015).

\section{Author contributions}

Y.Z. and M.Z. conceived and designed the experiments. Y.Z. and T.Z. performed the experiments. J.Q. and Y.W. analyzed the data. N.F. and C.M. provided the analysis tools. Y.Z. wrote the manuscript. All authors read and approved the final manuscript.

\section{Funding}

This work was financially supported by the National Natural Science Foundation of China (Grant Numbers 21966025 and 21667023), the Ningxia Key Research and Development Program (Grant Nos. 2019BFG02020), and the project of the Ministry of Education "ChunHui" plan (Grant Number Z2016068). The funders played no role in the design of the study and collection, analysis, and interpretation of data and in writing the manuscript.

\section{Competing interests}

The authors declare no competing interests.

\section{Additional information}

Supplementary Information The online version contains supplementary material available at https://doi.org/ 10.1038/s41598-021-04599-5.

Correspondence and requests for materials should be addressed to M.Z.

Reprints and permissions information is available at www.nature.com/reprints.

Publisher's note Springer Nature remains neutral with regard to jurisdictional claims in published maps and institutional affiliations. 
(c) (i) Open Access This article is licensed under a Creative Commons Attribution 4.0 International cc) License, which permits use, sharing, adaptation, distribution and reproduction in any medium or format, as long as you give appropriate credit to the original author(s) and the source, provide a link to the Creative Commons licence, and indicate if changes were made. The images or other third party material in this article are included in the article's Creative Commons licence, unless indicated otherwise in a credit line to the material. If material is not included in the article's Creative Commons licence and your intended use is not permitted by statutory regulation or exceeds the permitted use, you will need to obtain permission directly from the copyright holder. To view a copy of this licence, visit http://creativecommons.org/licenses/by/4.0/.

(C) The Author(s) 2022 